\title{
Viral Evasion of the Interferon System
}

\author{
Michael Gale Jr. ${ }^{1}$ and Ganes C. Sen ${ }^{2}$
}

$\mathrm{T}$ HIS SPECIAL ISSUE OF the Journal is dedicated to the topic of "Viral Evasion of the Interferon System"; it has been edited by Gale and Sen, and the individual articles have been contributed by authors who are experts on specific viruses and their host responses.

The actions of type I interferons (IFNs) provide our first barrier of defense against virus infection and are the foundation of innate immunity. Upon virus infection host pathogen recognition receptors, including the Toll-like receptors (TLRs), RIG-I-like receptors (RLRs), and/or various other factors including protein kinase R (PKR), 2'-5' oligoadenylate synthetase (OAS), and other nucleic acid-binding proteins that may serve as pathogen recognition receptors, function to discriminate self from nonself macromolecule ligands within the cell, and their engagement of nonself viral products triggers intracellular signaling cascades that drive the production of IFN and its secretion from the infected cell. The secreted IFN plays a major role in immunity by binding to the IFN receptors on the infected cell and on the neighboring bystander cells wherein signaling is initiated through the Jak-STAT pathway to drive the expression of hundreds of IFN-stimulated genes. It is the products of the IFN-stimulated genes (ISG) that confer the antiviral, immunomodulatory, and overall actions of IFN that limit virus replication and spread, and that enhance the adaptive immune response to infection. Curiously, along with IFNs, many of the IFN-stimulated genes are also induced directly by virus infection, probably to confer resistance to the infected cell itself.

Virus recognition as nonself occurs when a pathogen recognition receptor of the host cell may physically bind to a viral ligand. Virus infection typically triggers pathogen recognition receptor signaling and IFN production, though it is becoming increasingly clear that these processes and their outcome are cell-type specific in vivo, thus offering insights into the uniqueness of viral tropism and disease. In order to replicate and spread, viruses have to overcome local and even systemic IFN defenses, and virtually all pathogenic viruses have evolved the means to evade and/or suppress IFN defenses as a means of supporting virus growth. Many viruses encode pathogen recognition receptor antagonists and/or IFN antagonists within their genome. Such factors may direct blockades of intracellular signaling programs that otherwise trigger IFN production, confer signaling through the IFN receptor, or directly alter the function of IFN-stimulated gene products. Viral disruption of IFN production and action is tightly linked with virulence.

Understanding the molecular mechanisms by which viruses trigger and evade IFN actions are therefore of paramount importance for defining strategies aimed at controlling virus infection. The nature of viral pathogen-associated molecular patterns or the macromolecules that trigger IFN production is currently an intense area of study. While such work has shown that viral DNA or RNA can stimulate IFN production, it has also revealed that the exact nature of the pathogen-associated molecular pattern embedded within viral nucleic acid might be as diverse as the different viruses that trigger IFN production. Double-stranded RNA, B-form DNA, single-stranded RNA, polyuridine RNA, and various nucleic acid compositions have each been defined as pathogen-associated molecular patterns that are engaged by TLRs, RLRs, or other distinct pathogen recognition receptors. Ongoing and future studies should further define the nature of specific pathogen-associated molecular patterns within viral nucleic acid as well as viral proteins and virusassociated lipids.

Viruses are good at multitasking and they often impose multiple blockades within the infected cell to evade and limit IFN defenses. These range from disrupting TLR or RLR signaling to dysregulating Jak-STAT signaling and controlling ISG function. The use of multiple strategies to evade and counter IFN defenses could reflect the need to regulate innate immunity within a variety of different cell types as infection proceeds from the initial portal of entry to the target tissue/cell type of virus amplification in vivo. This underscores the distinctions by which various cell types recognize and respond to virus infection, whereby distinct cell types feature TLR versus RLR pathways of IFN production to express specific IFN- $\alpha$ subtypes and a cell-specific bioset of ISGs, possibly reflecting specialized organotypic functions of immunity.

An example of IFN as a major therapeutic for virus infection is found in the population of hepatitis $\mathrm{C}$ virus (HCV)infected patients. Pharmacologic preparations of IFN- $\alpha 2 \mathrm{a}$,

${ }^{1}$ Department of Immunology, University of Washington School of Medicine, Seattle, Washington.

${ }^{2}$ Department of Molecular Genetics, Lerner Research Institute, Cleveland Clinic, Cleveland, Ohio. 
IFN- $\alpha 2 b$, or consensus IFN, in conjunction with ribavirin, are the currently approved therapies for chronic HCV infection. Moreover, various forms of IFN are used as anticancer therapy and therapeutics for autoimmune diseases. Thus, an understanding of IFN actions, including mechanisms of viral and host response or resistance to IFN, is essential for refining and improving IFN-based therapy applications. In the example of $\mathrm{HCV}$, only about $50 \%$ of treated patients overall respond to IFN therapy or relapse after therapy cessation. In this case $\mathrm{HCV}$ is shown to suppress pathogen recognition receptor signaling in the infected cell, but it is not clear if the virus itself may truly evolve into an IFN-resistant variant during therapy. This underscores a need to fully define the mechanisms of viral evasion of IFN actions and to understand the many aspects of cross talk between various cellular processes/signaling pathways and IFN-responsive cellular programs that regulate infection and immunity.

In this issue, the topic of viral evasion of the IFN system has been broadly addressed by a number of leaders of the field; the authors have strived to provide a state-of-the-art picture, rather than a comprehensive historical account of the subject matter. However, for interested readers, the reference lists are inclusive enough to trace all relevant important observations by various investigators. The article by George and others focuses on 2 dsRNA-binding enzymes, PKR and ADAR1, which are important nodes of evasion by many viruses. Horner and Gale provide an introduction to the RLR pathways of cytoplasmic innate immune signaling in the context of HCV and discuss how this virus evades these pathways. Rieder and Conzelmann focus on 2 prototypic rhabdoviruses, vesicular stomatitis virus and rabies virus, and compare and contrast their distinct evasion strategies. The article by Basler and Amarasinhe deals with the evasion strategies used by filoviruses; specifically, the highly pathogenic Marburg and Ebola viruses. Diamond discusses how different flaviviruses antagonize the IFN system. Because much of our early knowledge of how RNA viruses evade innate immune responses of the host has come from investigations of the paramyxoviruses, 2 articles have been devoted to them. Ramachandran and Horvath discuss how these viruses disrupt IFN signaling by interfering with the actions of STAT proteins, whereas Goodbourn and Randall deal with viral blockage of IFN induction as triggered by the RLH pathways. Wolff and Ludwig provide a balanced account of various mechanisms used by influenza viruses to evade specific aspects of the IFN system. Moving on to double-stranded RNA viruses, Sherry tells us how rotavirus and reovirus deal with the IFN response. The article by Neil and Bieniasz focuses on retroviruses, more specifically HIV-1, and educates us about several distinct restriction factors of HIV-1 replication, their relationship to the IFN system, and how various viral proteins can antagonize them. The poxviruses employ a plethora of strategies to counteract both the innate and the adaptive arms of the immune system. In this context, Perdiguero and Esteban focus on the mechanisms used by vaccinia virus to evade the IFN system. There are 3 articles on herpesviruses: Paladino and Mossman tell us about the evading mechanisms used by herpes simplex virus-1, whereas Marshall and Geballe discuss multiple strategies employed by different cytomegaloviruses. Lee and others explore the world of viral IFN regulatory factors in the context of Kaposi's sarcoma-associated herpesvirus and tell us how they interfere with the IFN system and the P53 pathway. Finally, Beglin and others report how another clinically important virus family, the papilloma viruses, interfere with the functioning of the IFN system.

Address correspondence to: Dr. Michael Gale Jr.

Department of Immunology University of Washington School of Medicine 1959 N.E. Pacific Street, Box 357650

Seattle, WA 98195

E-mail: mgale@u.washington.edu

Or

Dr. Ganes C. Sen Department of Molecular Genetics Cleveland Clinic 9500 Euclid Avenue, NE20 Cleveland, $\mathrm{OH} 44195$

E-mail: seng@ccf.org

Received 29 June 2009/Accepted 1 July 2009 